\title{
Provision of coordinated care for individuals with Down syndrome: The Calgary perspective
}

\author{
Donna Heerensperger, RN, BScN \\ Clinical Resource Nurse, Down Syndrome Clinic, Alberta Children's Hospital, Calgary, Alberta, Canada
}

\begin{abstract}
In Calgary, Alberta, Canada, cooperation between families, agencies and health care providers has resulted in services that improve the health and quality of life for individuals with Down syndrome. One of these is the multidisciplinary Down syndrome team at the Alberta Children's Hospital, which provides assessment, treatment and support based on established the Down Syndrome Medical Guidelines (Cohen, 1999) to children with Down syndrome. Originally established to provide services to children from birth to six years of age, the clinic now sees children until the age of 18 years. This change in clinic mandate has enriched and changed the practice of the team. They have an increased awareness of how issues develop over time and impact the child's functioning and quality of life and have developed approaches to prevent and minimise these challenges. This article describes the Down syndrome team's evolution and the benefits of a multidisciplinary/coordinated approach for individuals with Down syndrome.
\end{abstract}

Key words: Down syndrome, Paediatrics, Calgary, Canada, clinics, case study, Community Health Services

\section{Down syndrome Clinic: The beginning}

In the late 1980s a group of Calgary parents, in conjunction with community agencies and the Alberta Children's Hospital $(\mathrm{ACH})$ brought together a team of professionals to provide care to children with Down syndrome using the Down Syndrome Medical Guidelines (Cohen, 1999) as a framework for care. The team consisted of a Developmental Paediatrician, a Clinical Resource Nurse (CRN), a Physiotherapist (PT), Occupational Therapist (OT) and Speech-Language Pathologist (SLP), and operated within the Preschool Developmental Clinic at Alberta Children's Hospital. The parent support group purchased a computer for the program and also provided funding to cover the cost of the nursing position. The initial pilot program involved 8 infants with Down syndrome and gradually the case load grew by approximately 12-13 infants a year.

In the early years, team members utilised their skills to address the child's needs. It was assumed that the child's medical needs were being addressed by the physicians involved, and the therapists focused on promoting skill development. The importance of regular medical follow up, and the use of the medical guidelines was encouraged, however, the responsibility for this was with the family, and contact between the team and physicians was primarily 'one-way', through written reports of therapy sessions.
The structure of the clinic meant that children were followed for a short time only. Upon reaching their sixth birthday, it was expected that therapy services would be accessed through the schools, and that community pediatricians would provide ongoing medical care as outlined in the Medical Guidelines (Cohen, 1999). It was recognised that often the transition was not smooth, and that not all children were able to access care; however, the Down syndrome team, as part of the Preschool Developmental Clinic was mandated to close files at the time of school entry. In view of these limitations it was determined that a change of our service delivery model was needed.

\section{Why was the Children's Hospital chosen as the site for this service?}

There were many advantages to having the team exist within the structure of ACH. The hospital's location, services and philosophy serve as the framework for the program.

The hospital provides service to Calgary area children from zero to 18 years of age as well as those who travel from southern Alberta, southeastern British Columbia and southwestern Saskatchewan. It is the only freestanding, exclusively paediatric facility and research centre between British Columbia and Winnipeg.

At the Alberta Children's Hospital, a philosophy of familycentred care is promoted. It is recognised that children 
Down syndrome team case load

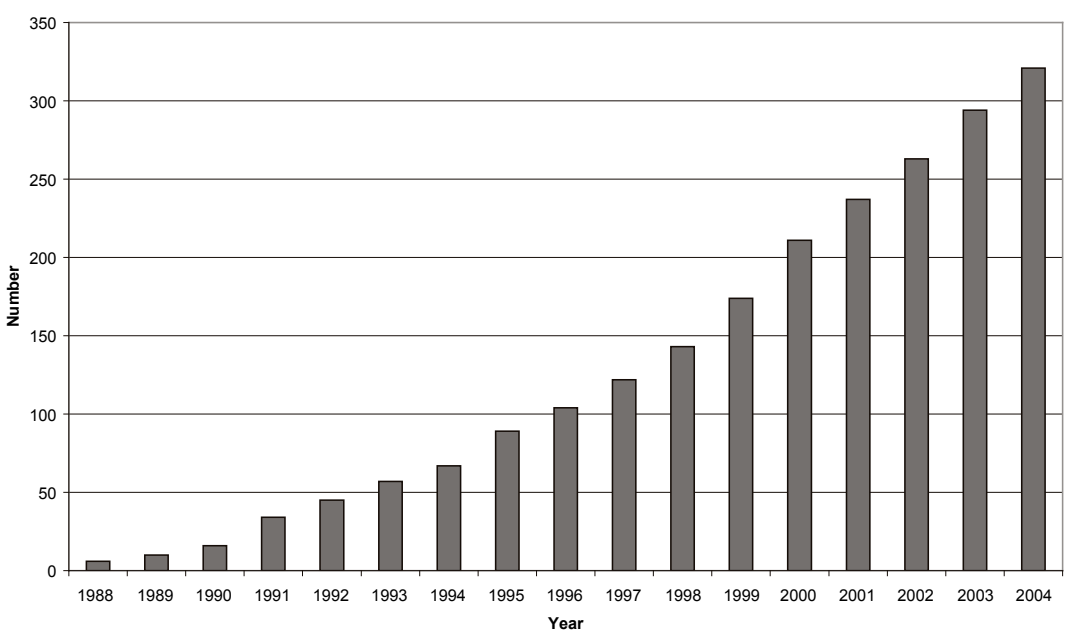

Figure I. Clinic case load

often heal better in their own home environment. That is why many of the patients are seen as outpatients. In other words, they come in for appointments with the specialists and health care teams and return home at the end of their visits. These outpatient clinics include: Asthma, Cardiology, Paediatric Dentistry, Developmental (including the Down Syndrome Team), Diabetes, Endocrine, Gastrointestinal, Haematology, Infectious Disease, Nephrology/Urology, Neurology, Neurosurgery, Oncology, Orthopaedic, Pulmonary, Refractory Epilepsy, Respiratory, Rheumatology, Sleep Clinic, Vision and Hearing.

In addition to caring for the many outpatients, the Alberta Children's Hospital has approximately 110 beds for children who require both short and long term stays in the hospital. Families are important members of the team in promoting health and wellness to children and youth.

A supervised, play/waiting area for $\mathrm{ACH}$ patients and their siblings is available to support children and their families.

Down Syndrome Reassessments

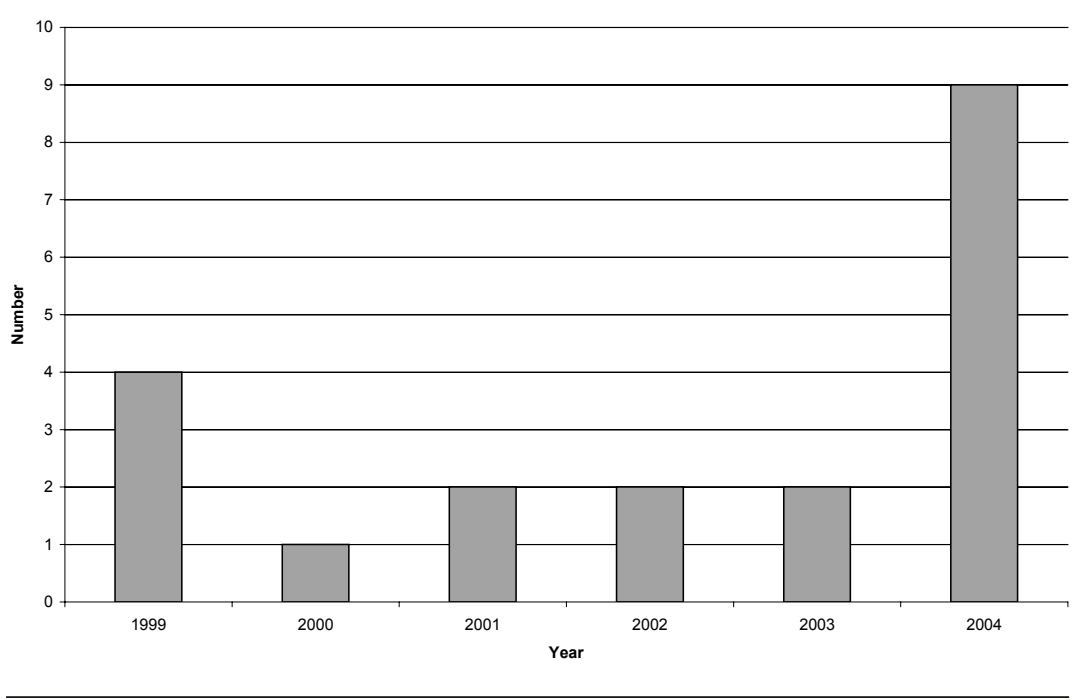

This area is staffed by volunteers and there is no charge for this service.

Parent Services advisors are also available for families at the hospital for both outpatient and inpatient areas. Their role is to spend time with families, help reduce worries, and provide general information and support. They also have books and brochures available on a variety of topics relating to children's health, and a 'parent's room' where families can breastfeed their infant, settle them for a nap, or take a 'breather' from appointments.

Another hospital resource is the Multicultural Committee which ensures quality care to families of diverse cultural backgrounds. Interpretive services are available. The use of traditional practices may be considered in consultation with the child's physician.

The location of the Down syndrome clinic within $\mathrm{ACH}$ enables families to access these resources and have appointments coordinated so that less time is spent away from home. Also, having these services under one roof allows the specialists to meet to discuss the child's care, promoting seamless service.

\section{What changes have occurred over time?}

\section{Growth}

Reorganisation of hospital services in the 1990s brought about changes - most importantly, the extension of the team's mandate to 18 years of age. Rather than discharging children from the clinic, the team had the mandate to continue to follow them into adolescence.

With the increase in mandate, the clinic case load has risen to over 350 children (see Figure 1). As the city has grown, the number of births has risen from $12-13$ a year to $25-26$, and an increasing number of older children are being sent back to clinic by their primary physicians (Figure 2). As time passes, physicians from outlying areas are sending an increased number of referrals as well, often urged on by parents or community therapists who have heard of the program. This growth brings a further challenge as team staffing levels have not risen in proportion to the caseload (Figure 3).

\section{Figure 2. Down syndrome reassessments}




\section{Staffing}

Over the years, the team has been fortunate to attract additional professionals to provide care to children with Down syndrome. This includes a Social Worker, Audiologist, Clinical Dietitian, and Ear/ Nose/Throat specialist.

In order to maximise limited resources and be responsive to the community, the linkages with the Down syndrome support group have been maintained and strengthened over the years. Decisions about staffing, mandate, and inclusion/exclusion criteria, for example were made in consultation with the clinic's community partners, the support group, and families.

The support group also provides funding to allow the Clinical Resource Nurse to work a second day a week, and have been generous with funding to send team members to the Canadian Down Syndrome Society Conference on a yearly basis. They have provided funds to purchase toys and equipment for the clinic, and supply the materials for heel cups so that they can be offered to families free of charge.

\section{Treatment challenges:}

Expanding service delivery to eighteen years of age revealed issues and concerns that the team had never dealt with before. Children were being referred because of behavioural and learning issues in the home and classroom, problems with sleep and toilet training, issues related to nutrition, weight gain, puberty, social concerns, and unresolved feeding issues. The team were fortunate to have access to an enormous amount of research on Down syndrome, child health and development. It was recognised that in addition to identifying and treating the problems, it was also necessary to look at ways to prevent the problems from arising, to provide education and support to families, agencies and schools, and to educate other service providers. As members of the Down syndrome team, therapists began to gain experience and confidence in providing consultation to this population.

One of the most significant lessons learned was that a person's understanding of the impact of extra $21^{\text {st }}$ chromosomal material is often based on misinformation or preconceived ideas. The Surgeon General's report (2001) referred to it as "diagnostic overshadowing" and commented that it continues to limit access to appropriate and quality care. The service team has learned that utilising the Health Guidelines (Cohen, 1999) does not always guarantee that issues are being identified and treated appropriately. If the individual believes that people with Down syndrome are 'slow', have poor muscle tone, are often obese, have constipation
Down Syndrome Referral rate

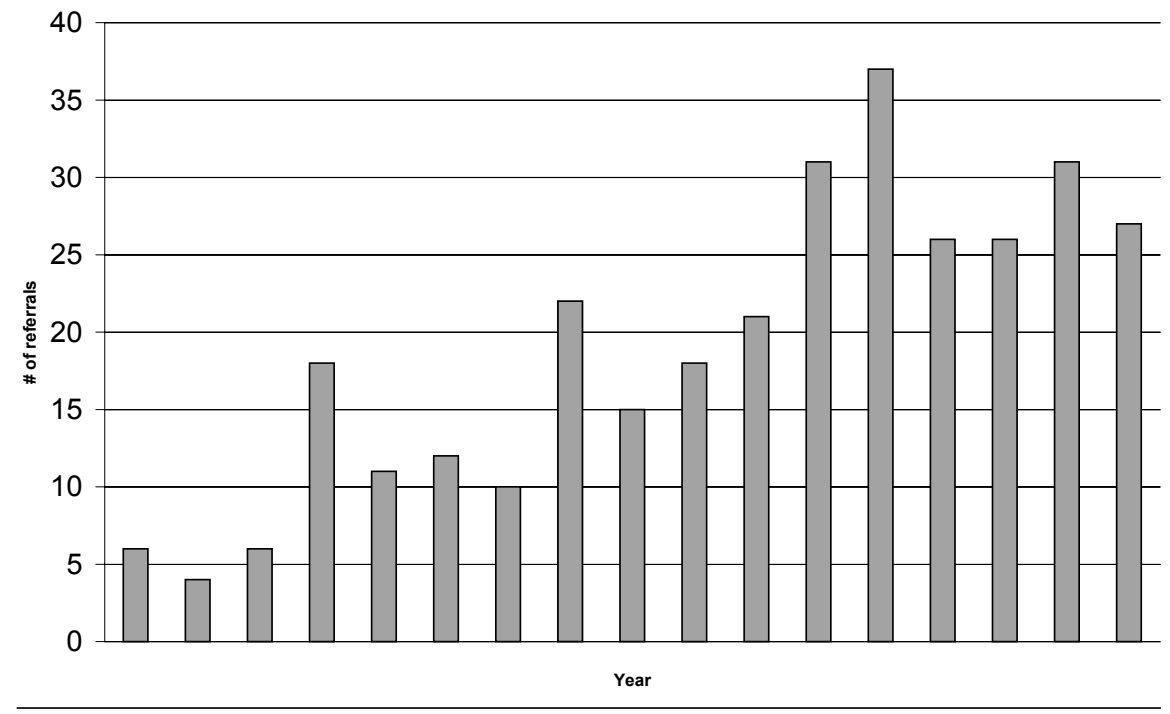

Year

and feeding challenges, learning, behaviour and attention difficulties, sleep poorly and snore, have poor vision and hearing, and will eventually develop Alzheimer Disease, they may not look beyond the Down syndrome to identify the cause and treat it.

If these issues are considered 'normal' for people with Down syndrome, they are often not investigated and can impact on every aspect of the individuals' and families' lives. Families have reported that when they have suggested that intervention was needed, they were often identified as "unrealistic" or "in denial".

Staff at the clinic have often found that health issues play a role in the problems that bring the children to us. Children who have been referred with behavioural concerns have made significant changes when previously undiagnosed Obstructive Sleep Apnoa has been identified and treated; when children with undiagnosed severe hearing loss have been provided with hearing aides, and when suggestions have been made to increase a child's 'core strength' when his or her low tone prevented him/her from sitting still in the classroom. Children who have lived with constipation for years have been diagnosed with Hirschsprung Disease, Hypoactive Thyroids, or been given strategies to manage their bowels successfully.

Experience has shown that, prior to any Developmental or Behavioural assessments, it is necessary to rule out possible health problems. To this end, a complete nursing assessment is completed by telephone when a child is referred to clinic. This assessment reviews the child's health history, parental/school concerns, status of body systems (cardiac, respiratory, endocrine, digestive, immunologic, neurological, urologic, growth, general health, etc). Information about the family, community and resources is obtained in order to ensure that appropriate therapies and supports are in place. Based on the information obtained, a plan is developed with the family, and a package is mailed to them 
which includes consents, history forms, a Minnesota Infant Development or Child Developmental Inventory (see glossary) (Doig, et al, 1999), as well as information about the clinic and community resources.

When the signed parent consents have been received, it is possible to access information from the birth hospital, community treatment programs and schools. This information assists the team in identifying areas of concern, and planning their session to meet the needs of the child, family and community. Experience has shown that these reports can reveal surprises: an echocardiogram that was not done; an abnormal echocardiogram that parents thought was normal; thyroid function tests that were not done; hearing reassessments that were missed, etc.

Experience brought increased confidence and the team began to advocate more effectively for the children in their care. Team members began to build linkages with other outpatient clinics, and utilise their expertise when problems came up.

Rather than relying on families to communicate with their physician, or that the physician would read our clinic notes, the team began to contact physicians directly when concerns arose. Soon physicians were calling team members directly as well to consult about specific issues. Rather than separate individuals working in isolation, staff began to see that working together as a team was more effective.

People with Down syndrome are complex, and need a team approach. This team ideally includes parents, family, caregivers, teachers, physicians, therapists, and community programs. The ability to meet as a group - whether it is face to face, via teleconference, videoconferencing - brings many benefits. These include a healthier child who thrives with appropriate interventions, treatments and program planning; a stronger support network for the child and family as they learn about the child's needs and the resources they can use to meet them; and a resilient family who can articulate their concerns and advocate successfully.

\section{Case study}

B.S. is an infant boy with Down syndrome, who lives in another Alberta city. His paediatrician, with encouragement from B.S.'s treatment team and his mother, referred B.S. to the Down Syndrome Team at Alberta Children's Hospital when he was 14 months of age. The referral did not specify specific concerns, other than the fact that B.S. had Down syndrome.

Prior to the Clinical Resource Nurse contacting the family, the physiotherapist from B.S.'s treating team contacted the nurse directly to discuss her concerns. She indicated that according to parent report, "B.S. is able to roll; however, she has never seen this. He has very low tone, doesn't reach, and primarily sucks his fingers. The team has concerns about his vision as well and feels that he doesn't appear to process what he sees, and they are unsure if his vision had ever been checked. They also question his hearing and cardiac status. B.S. is able to drink from a spouted cup". The team had consent from B.S.'s family to send the Down Syndrome Team all available documentation, and was requesting a videoconference when our assessments were completed.

The Clinical Resource Nurse contacted the child's mother (C.S.) by phone to discuss her concerns. C.S. indicated her biggest concern was B.S.'s delayed development despite good health.

B.S. is the first child of C.S. and T.S. T.S. has 39 genetic anomalies, but has no definitive diagnosis. The anomalies include a missing kidney, Simian creases on palms and feet, low set ears, 8 toes, small cheek bones and a long tailbone. $\mathrm{He}$ also has Hypospadius, and learning difficulties. He is currently in College and doing well. At 16 weeks gestation, C.S. experienced bleeding, and an ultrasound was suggestive of Down syndrome, which was confirmed via amniocentesis. B.S. was born via spontaneous vaginal delivery at 34 weeks gestation, and went to the Intensive Care Unit because of prolonged rupture of membranes.

B.S. was reported to be healthy, with no allergies. He was taking a multivitamin and his immunisations were up to date. He has never received influenza vaccine. In the past, the family had tried Mineral oil and Lactulose to treat B.S.'s chronic constipation, but was now trying Catnip and Fennel in a glycerin base. He was given $2 \mathrm{ml}$ from a dropper at every meal and $60 \mathrm{ml}$ of Alovera Juice $60 \mathrm{ml}$. in his juice at mealtime. B.S.'s constipation has been a significant issue since solid food was introduced, and has been associated with rectal bleeding.

B.S. had an echocardiogram at nine months of age and was diagnosed with an Atrial Septal defect. C.S. understood that this would not have an impact on B.S.'s development, but was unsure about if and when follow up should occur. B.S.'s thyroid and blood count status was unknown, and by parent report they had not been checked since birth. There were no parental concerns about B.S.'s respiratory status, although it was noted that he appeared 'bluish' around his eyes while sleeping. Vision and hearing had been screened at a year of age, however, audiology results were 'inconclusive', and although a 'lazy right eye' was noted, no follow up was planned. B.S. had a circumcision done as a day surgery at the local hospital, and has had no other surgeries. By parent report, B.S. was at the 75 th percentile for height and 50th percentile for weight on the Down Syndrome Growth Chart.

B.S. slept through the night at 3 months of age and typically sleeps with his back arched, head thrown back, or on his stomach or side. His mother reports that B.S. gets "hiccups" at least 6 times in 24 hrs. When asked to describe the "hiccup" she reported that if he is sitting up, he falls forward, eyes rolls back - describing it as a jolt. He then pulls back up and screams. If he is lying down, he gets "the same facial expression", his arms jolt forward a bit, eyes roll up, grimace on his face. Other times he will have one "hiccup" then does "little jerks". These episodes occur first thing in the morning, after his morning and afternoon naps, and wake him up about three times a night. C.S. had reported 
these events to B.S.'s paediatrician as well as his cardiologist and had been reassured.

B.S.'s diet consists of Cream of Wheat with fruit for breakfast, 3 or 4 vegetables with chicken at lunch, sometimes rice, and supper is usually roast beef or chicken. Purees were introduced at six months of age and C.S. continues to puree all of B.S.'s food, as she reports that he chokes easily and doesn't chew at all. He sucks the food to the back of his throat so he can swallow it. He refuses Cheerios (chokes on an 8 th of one) but can eat orange sections if the skin is removed, or Ichiban noodles if they are chopped.

B.S. was a "messy" breast feeder, and often chokes on liquids, especially if they are given by spoon. Despite the choking episodes, C.S. feels that B.S. can adequately protect his airway, and reports no history of respiratory illnesses or pneumonia.

B.S. is not an independent feeder as he is not yet reaching for objects, and has recently started to poke at his food while being fed. The only thing he brings to his mouth is his hands and sucks on his fingers while being fed to help move the food back in his mouth.

B.S.'s central lower incisors erupted at fifteen months of age, and he appears to be teething again. He gets mad when his mother goes near his face with a toothbrush and will only open his mouth to a spoon.

B.S. receives bi-weekly therapy services in the home from Occupational Therapy, Physiotherapy and Speech-language Pathology. Although the family is eligible for special needs funding support, they have not accessed this resource. They have limited opportunities to access respite, due to financial restraints, and B.S.'s high needs, although family members will assist occasionally.

Based on the phone conversation with C.S., the following issues were identified:

1. "Hiccups" - are these infantile spasms?

2. Developmental delay - is this reflective of more than Down syndrome? Are the "hiccups" related?

3. Feeding/swallowing/chewing difficulties - at risk for aspiration?

4. Vision status.

5. Hearing status.

6. Health status - needs blood work as per the Down Syndrome Medical Guidelines, including CBC and Free T4, TSH.

7. Cardiac status - need to review documentation to determine need for further follow-up.

8. Herbal remedies - are they safe and appropriate?

9. Chronic constipation.

10. Possible Obstructive Sleep Apnœa - unusual sleep position, blueness around eyes, developmental delay, restless sleep.

The following plans were made in consultation with the family:
1. Clinical Resource Nurse advised C.S. that her report of "hiccups" was of concern, and further investigation of these events needed to be done. The Clinical Resource Nurse will review the information with the Developmental Paediatrician and will contact referring physician with our concerns and suggestions. Family in agreement with referrals to Neurology clinic, Ear Nose and Throat Clinic (ENT), Gastroenterology, Surgical, Dentistry and Sleep Clinic if Developmental Paediatrician feels appropriate.

2. Clinical Resource Nurse will contact B.S.'s treating team about "hiccups" to determine if they have witnessed them and ask them to follow up.

3. Clinical Resource Nurse will arrange for Social Work, Developmental Paediatrics, Ophthalmology, and Audiology appointments as soon as possible, as well as Neurology, Sleep Clinic, ENT, and Gastroenterology if Developmental Paediatrician in agreement.

4. Following medical work up, arrangements will be made for a feeding and developmental assessment with Down Syndrome Team Occupational Therapist, Physiotherapist, Speech Language Pathologist.

5. C.S. will advise Clinical Resource Nurse if the family will need accommodation at Ronald McDonald House. At this time, they prefer to stay with extended family in the city.

After reviewing the above information with the Developmental Paediatrician, the appointments and referrals were arranged as per the above plan. Over the next few months, this family made numerous trips to Calgary for assessments and follow-up. Neurology saw B.S. on an urgent basis, an EEG was done, and the "hiccups" were diagnosed as Infantile Spasms. A short time later, partial seizures were noted as well. An MRI under general anæsthetic showed delayed mylenation. Controlling these events has proven to be extremely difficult, and although parent report indicated an improvement, the EEGs did not change.

Currently, B.S. is undergoing a trial of ACTH (see glossary) and a follow up EEG has demonstrated a response to the drug. He will continue to receive injections every other day, and will then be weaned off over a three month period. Recently, B.S.'s family and treating team noted that he was exhibiting autistic-like behaviours and is increasingly irritable and difficult to console. Eisermann, DeLaRaillere et al. have reported a statistically significant correlation between treatment lag and lag to cessation of spasms, developmental quotient and score of autistic features. They found that the later the response to treatment of infantile spasms, the lower the developmental quotient and the higher was the score of autistic features. A long duration of spasms also determined a low developmental quotient and a high score of autistic features. Resources have been put in place to increase this family's access to respite, and strategies to manage B.S.'s behaviours have been developed.

B.S. was hospitalised in his local hospital with pneumonia shortly after the telephone interview, therefore a Videofluoroscopy was arranged on an urgent basis and confirmed that 
B.S. is a silent aspirator. A Nasogastric tube is now utilised for fluids, and oral feeding is limited to thick purees. His parents have decided to pursue a Gastrostomy, however, further testing to rule out Gastroesophageal Reflux will be done prior to this surgery. This will necessitate an inpatient admission, as he will need to have his Nasogastric tube removed for 24 hours prior to the $\mathrm{x}$-rays and will require intravenous fluids. Given his immune-compromised status, this will be postponed until his course of ACTH is finished. If reflux is identified, B.S. will have a Fundoplication done at the same time as his Gastrostomy.

Hirschsprung Disease was ruled out with a rectal biopsy; however B.S. was diagnosed with bilateral undescended testes. Surgery is planned for a later date, following stabilisation of his seizures and feeding issues.

B.S.'s parents received some positive news as well. Although B.S.'s screening for sleep apnœa (pulse oximetry) raised concerns, his polysomnography was normal, ruling out Obstructive Sleep Apnœa. His vision and hearing were within normal limits, and will continue to be monitored on a regular basis.

B.S.'s TSH was found to be $6.34 \mathrm{mU} / \mathrm{L}$ and Free T4 14 $\mathrm{mU} / \mathrm{L}$, and so he was referred to the Endocrine Clinic, who felt the results were influenced by B.S.'s seizure medications, and will continue to monitor his thyroid status.

Review of B.S.'s cardiology consultation report confirmed that B.S. has an Atrial Septal Defect, and suggested follow up by a cardiologist should be at the physician's discretion. No antibiotic prophylaxis is indicated.

Independently, B.S.'s parents decided to stop using herbal therapies, as they did not feel they were beneficial in addressing the constipation issue. C. S. was reviewed by Paediatric Dentistry and was found to have no caries.

The Down syndrome team completed developmental assessments and provided the treating team and family with information and suggestions. B.S. is presenting with significant delays in all areas of his development, as well as some autistic-like features. Further monitoring and assessment (particularly when the seizure activity and aspiration issues are resolved) will hopefully assist in determining what the future will hold for B.S.

The use of live videoconferencing has allowed the family and involved professionals to meet 'face to face' to share concerns, ask questions, and plan care over a long distance. The result is less travelling for the family, and increased knowledge and confidence for the professionals. The long term goal is that B.S. (as well as other children with Down syndrome) is able to access the resources they need in their own community.

B.S.'s experience highlights the complexity of health issues that people with Down syndrome may have and the importance of looking beyond the diagnosis of Down syndrome. Although B.S. was living in a metropolitan area and accessing medical and therapeutic specialists, a number of health issues were unidentified which impacted not only B.S.'s health, progress and prognosis, but his family as well.

\section{Future directions}

Currently, only individuals under 18 years of age with Down syndrome can access the team's services. There is a high need for an adult clinic to provide coordinated care, and support individuals and their families as they move from paediatric services to the adult world. The development of an Adult Clinic would also provide professionals with the opportunity to develop expertise about adult issues, and perhaps strategies to reduce the problems through intervention earlier in the lifespan. At this time, the Adult Clinic remains a dream, but not an impossible one.

\section{Glossary}

ACTH: Also known as Prednisone, Cortrosyn. These are anti-inflammatory, steroid medications that are used to treat a number of neurological conditions including infantile spasms, Landau-Kleffner syndrome and certain cases of Lennox-Gastaut syndrome.

Minnesota Child Developmental Inventory (CDI): This tool is completed by the parent and measures the child's development in eight areas: social, self help, gross motor, fine motor, expressive language, language comprehension, letters, and numbers. It also includes a General Development Scale and 30 items to identify parents' concerns about their child's health and growth, vision and hearing, development and behaviour.

Minnesota Infant Development Inventory (IDI): This tool is completed by the parent and measures the infant's development in five areas: social, self-help, gross motor, fine motor and language. Parents can also report any questions or concerns about their baby's health, development, or behaviour.

\section{Correspondence}

\section{Donna.Heerensperger@CalgaryHealthRegion.ca}

\section{References}

Cohen, W.I. (Ed.). (1999). Health Care Guidelines for Individuals with Down Syndrome, Down Syndrome Quarterly, 4(3), 1-16.

U.S. Public Health Service. Closing the Gap: A National Blueprint for Improving the Health of Individuals with Mental Retardation. Report of the Surgeon General's Conference on Health Disparities and Mental Retardation. February 2001. Washington, D.C., page A-5.

Doig, K.B., Macias, M.M., Saylor, C.F., Craver, J.R. \& Ingram, P.E. (1999). The Child Development Inventory: A developmental outcome measure for follow-up of the high-risk infant. Journal of Pediatrics, 3, 358-62.

Eisermann, M.M., DeLaRaillère, A., Dellatolas, G., Tozzi, E., Nabbout, R., Dulac, O. \& Chiron, C. (2003). Infantile spasms in Down syndrome - effects of delayed anticonvulsive treatment. Epilepsy Research, 55, 21-27. 